\title{
Processo de Calibração dos Microparâmetros em Método de Elementos Discretos via Generalized Simulated Annealing
}

Felipe Tajá Costa Pinto ${ }^{\star 1,2}$, Raquel Quadros Velloso $1,{ }^{1}$ PUC-Rio, ${ }^{2}$ Petrobras

Copyright 2021, SBGf - Sociedade Brasileira de Geofísica

This paper was prepared for presentation during the $17^{\text {th }}$ International Congress of the Brazilian Geophysical Society held in Rio de Janeiro, Brazil, 16-19 August 2021.

Contents of this paper were reviewed by the Technical Committee of the $17^{\text {th }}$ International Congress of the Brazilian Geophysical Society and do not necessarily represent any position of the SBGf, its officers or members. Electronic reproduction or storage of any part of this paper for commercial purposes without the written consent of the Brazilian Geophysical Society is prohibited.

\section{Abstract}

The Discrete Element Method (DEM) is a numerical computational technique that simulates the macroscopic material behaviour by solving the equations of motion of its constituents. For a correct prediction of this behaviour, are set as input data the mechanical characteristics of the elements, the so-called microparameters. However, there is no recipe for determining these microparameters based solely on the macroscopic responses of the simulated material. It is required an additional step known as Calibration. The method widely used in this calibration is Trial and Error, although is an inefficient method due to its unfavorable scale factor. This work proposes a new approach using the Generalized Simulated Annealing global optimization method, minimizing the normalized quadratic area between the experimental and calculated curves of the axial stress-strain and volumetric-axial strains curves simultaneously. Comparison is done using triaxial tests for synthetic data whose results demonstrate the usefulness and applicability of the proposed approach.

\section{Introdução}

A simulação está presente em praticamente todos os campos do desenvolvimento humano e pode ser de elaborada de diversas maneiras. Para a simulação de mecânica de rochas ou materiais sólidos, existem algumas técnicas que são bastante empregadas para a avaliação de relações de tensão-deformação.

Para a simulação mecânica de rochas ou materiais sólidos existem algumas técnicas que são bastante empregadas para a avaliação de relações de tensão-deformação e/ou suas relações com o a propagação de ondas mecânicas. Entre elas estão o Método de Elementos Finitos (Finite Element Method - FEM), além de diversas técnicas sem uso de malhas (meshless ou mesh free) que tratam o material como um corpo contínuo. Contudo estas aproximações contínuas não conseguem reproduzir, de maneira simples, a ocorrência de fraturas que envolvem altas quantidades de descontinuidades. O Método de Elementos Discretos (Discrete Element Method - DEM) pode ser uma alternativa para estudos deste tipo de fenômeno, primariamente por levar naturalmente em conta as descontinuidades.

O Método dos Elementos Discretos é capaz de simular o comportamento macroscópico de um material via solução das equações do movimento de seus constituintes. Tratase de um método bastante versátil, que pode ser utilizado nos mais variados campos da Ciência e Engenharia como, por exemplo, em modelagens de ancoragem em solos, ensaios mecânicos e avaliação do comportamento de rocha dura, simulação de breakouts em poços, estudos de fluxo de fluidos em meios porosos e propagação de ondas mecânicas e de calor.

O DEM consiste em solucionar as equações do movimento para cada constituinte, avaliando o comportamento de todo o material. As forças entre os elementos são dadas por um modelo de contato e são estes modelos que descrevem a coesão do material.

Diferentemente dos métodos contínuos, no DEM é necessário incluir os parâmetros que descrevem a interação em microescala, os chamados microparâmetros, entre os seus elementos. Cada um deles manifesta uma macro-resposta distinta e, de maneira geral, não é possível se expressar, por uma única equação, a relação direta entre os microparâmetros à sua resposta macroscópica que se aplique a todos os casos, geometrias e modelos de contato. Desta maneira um procedimento inicial inconveniente, porém de grande importância deve ser efetuado: a calibração.

A calibração é um processo lento e computacionalmente dispendioso cuja técnica mais comumente utilizada é a conhecida como Tentativa e Erro (CHAREYRE et al, 2002), (LEE et al, 2016), (SCHOLTÈS \& DONZÉ, 2013). A fim de reduzir o tempo gasto nesta etapa, se faz necessária algum tipo de automatização. Uma forma de se realizar esta automatização é usando algum método de otimização, ou seja, de minimização de uma função objetivo.

\section{Teoria}

\section{Método de Elementos Discretos}

O Método de Elementos Discretos foi proposto originalmente por Cundall e Strack (1979) e consiste em representar o material como sendo composto de pequenas unidades interagentes que são chamadas de elementos discretos.

De uma maneira geral o DEM envolve cinco etapas:

1. Inicia-se um ciclo com a determinação do passo de tempo para aplicação das Leis do Movimento. Este passo de tempo pode ser dinâmico, obtido a partir do cálculo de propagação de onda compressional; ou estático, um valor fixo em toda a simulação;

2. Aplicação das Leis do Movimento, que, neste caso, é a segunda Lei de Newton, em cada um 
dos elementos do modelo. Este passo é realizado tanto para a translação quanto para a rotação;

3. Acréscimo do passo de tempo;

4. Detecção dos contatos, sendo, normalmente, um dos passos mais custosos computacionalmente;

5. Uma vez determinadas as novas posições, calculam-se as novas forças sobre cada elemento.

Este ciclo se repete até que um critério de parada seja atingido. A Figura 1 representa um ciclo de cálculos básico em DEM.

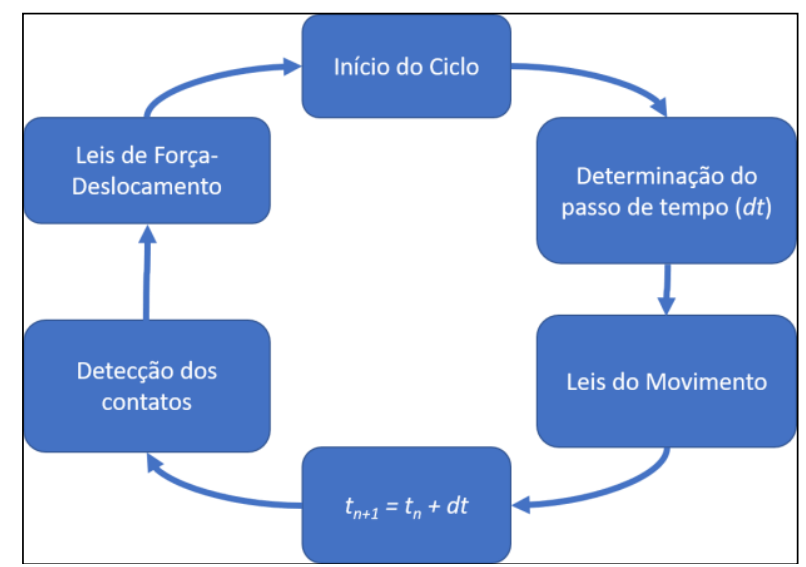

Figura 1: O esquema geral de cálculo em DEM.

A força entre os elementos é decomposta em duas direções: normal e cisalhante. Na direção normal a força é dada pela equação

$$
F_{n}=k_{n} u_{n}
$$

onde $k_{n}$ é a rigidez normal e $u_{n}$ é o deslocamento normal entre dois elementos interagentes. $\mathrm{O} k_{n}$ ainda pode ser escrito como

$$
k_{n}=E_{m} \frac{r_{1} r_{2}}{r_{1}+r_{2}}
$$

onde o termo $E_{m}$ é chamado de módulo elástico. Sob tração a ruptura acontece quando a força atinge o valor máximo dado por

$$
F_{n, m a ́ x}=-t A_{\text {contato }},
$$

onde $t$ é chamado de resistência à tração e $A$ é área de contato. A partir do momento que há a ruptura, a força normal é zerada.

A Figura 2 mostra as forças normais entre dois elementos.

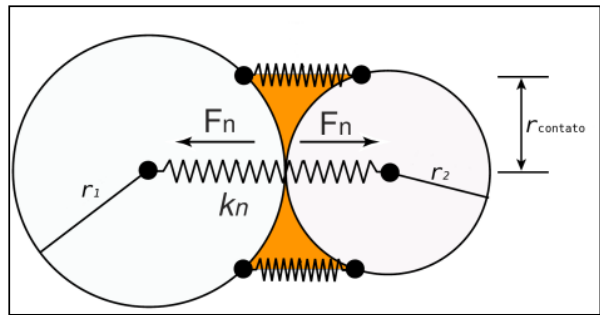

Figura 2: Representação das forças normais.

A força cisalhante total, que pode ser vista na Figura 3, é calculada de maneira incremental, atualizando a sua orientação e intensidade por um incremento da força cisalhante, ou seja,

$$
F_{s}=\left\{F_{s}\right\}_{\text {anterior }}+k_{s} \Delta u_{s}
$$

com $k_{s}$ sendo a rigidez cisalhante e $\Delta u_{s}$ é deslocamento tangencial incremental relativo.

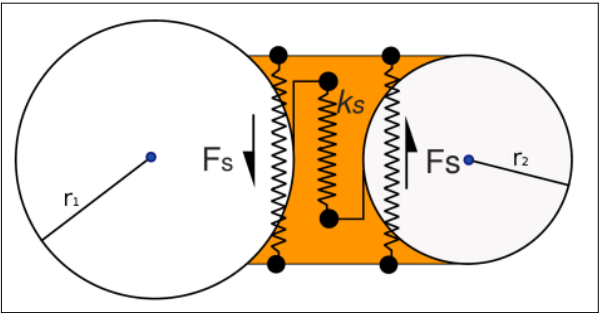

Figura 3: Representação das forças cisalhantes.

Para modelar o comportamento não linear da relação entre tensão-deformação, utilizou-se um modelo de MohrCoulomb modificado. A força de cisalhamento máxima é dependente da força normal $\left(F_{n}\right)$, coesão $(c)$, ângulo de atrito local $\left(\varphi_{b}\right)$. A força de cisalhamento máxima é calculada de acordo com a equação

$$
F_{s, \text { máx }}=F_{n} \tan \varphi_{b}+c A_{\text {contato }}
$$

Após a ruptura, os contatos são considerados puramente friccionais.

\section{Generalized Simulated Annealing}

O Generalized Simulated Annealing (TSALLIS \& STARIOLO, 1996) tem atraído uma relativa atenção por ser adequado em tratar problemas de minimização quando estão envolvidas muitas variáveis e o mínimo global está compreendido em um conjunto com vários outros mínimos de menor profundidade. As principais características que contribuem para este fato são: ser derivative free, a sua relativa facilidade de incorporação em códigos que necessitem de uma otimização; e pela capacidade de ser empregado com basicamente nenhuma informação sobre a natureza específica do problema.

De uma maneira abrangente, pode ser sumarizado da seguinte maneira:

1. Partindo de um ponto, um novo ponto será dado a partir da adição de um incremento aleatório $\delta x$. Com este novo ponto, calcula-se a função objetivo e se efetua a comparação com o valor no ponto anterior; 
2. Uma vez que esta diferença seja negativa, o movimento é aceito retornando-se ao processo. Se a diferença for positiva, o movimento pode ou não ser aceito, conforme um certo critério de aceitação;

3. Após uma quantidade determinada de vezes que este processo é repetido, reduz-se a temperatura.

Neste contexto, a temperatura é uma medida da intensidade da aleatoriedade do sistema, ou seja, à medida que o método evolui, a temperatura reduz, diminuindo a sua aleatoriedade. O fluxograma de todo este processo pode ser visto na Figura 4.

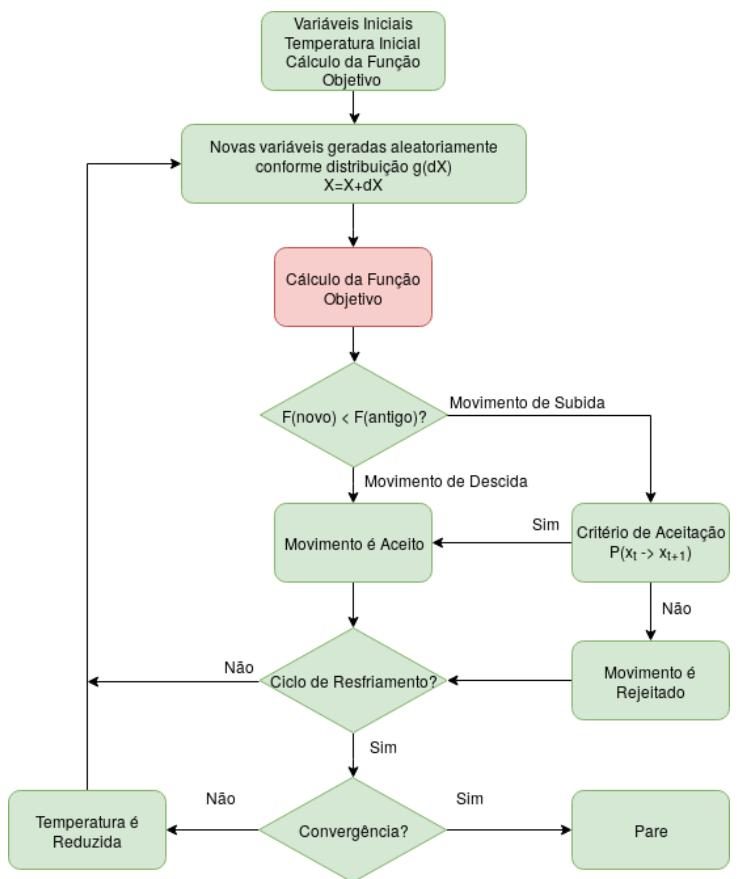

Figura 4: Fluxograma do GSA. A série de cálculos efetuados com uma mesma temperatura é chamada de cadeia de Markov.

A equação que rege a distribuição de probabilidade para o sorteio das novas variáveis tem 0 aspecto de uma distribuição de Cauchy-Lorentz distorcida. É determinada principalmente pelo parâmetro $q_{v}$ e sua expressão analítica é dada por

$$
\begin{gathered}
g_{q_{V}}(\delta x)=\left(\frac{q_{V}-1}{\pi}\right)^{D / 2} \frac{\Gamma\left(\frac{1}{q_{V}-1}+\frac{D-1}{2}\right)}{\Gamma\left(\frac{1}{q_{V}-1}-\frac{1}{2}\right)} \\
\left\{1 T_{q_{V}}^{V}(t)\right]^{-\frac{D}{3-q_{V}}} \\
\left\{1+\left(q_{V}-1\right) \frac{(\delta x)^{2}}{\left[T_{q_{V}}^{V}(t)\right]^{-\frac{2}{3-q_{V}}}}\right\}^{\frac{1}{q_{V-1}+2}}, \ldots \ldots \ldots . .
\end{gathered}
$$

onde $D$ é o número de variáveis utilizadas na busca, $q v$ é um parâmetro que controla a abertura da função e $T$ é a temperatura.
À medida que o algoritmo evolui, reduz-se a temperatura conforme a função definida por

$$
T_{q_{V}}^{V}(t)=T_{0} \frac{2^{q_{V}-1}-1}{(1+t)^{q_{V^{-1}}-1}}, \ldots \ldots \ldots(7)
$$

onde $T_{0}$ é a temperatura inicial e $q v$ define o quão rápido será esta redução da temperatura com o tempo, de maneira a garantir a convergência.

Para haver a possibilidade de fuga de mínimos locais, deve-se haver passos em que a função objetivo aumente em relação ao valor anterior. Para isto é preciso que a probabilidade de aceitação que seja dada pela expressão

$$
P_{q_{\mathcal{A}}}\left(\vec{r}_{t} \rightarrow \vec{r}_{t+1}\right)= \begin{cases}1 & , \text { se } \Delta f<0 \\ \frac{1}{\left[1+\left(q_{\mathcal{A}}-1\right) \frac{\Delta}{\tau_{q_{A}}^{t}}\right]^{1 /\left(q_{\mathcal{A}}-1\right)}} & , \text { se } \Delta f \geq 0\end{cases}
$$

onde $q_{A}$ é um parâmetro que regerá o tipo de probabilidade de aceitação utilizada.

O critério de aceitação funciona da seguinte forma: sorteiase um valor entre 0 e 1 com distribuição uniforme e compara-se com o valor calculado pela Equação 8. Caso o valor sorteado seja menor que $P_{q A}$, o passo de subida é aceito, caso contrário, descarta-se.

\section{Funcão Objetivo}

Todos os trabalhos encontrados na literatura que envolvem calibração e DEM realizam-se várias simulações dos experimentos para encontrar os macroparâmetros desejados. Por exemplo, simulam-se ensaios de compressão e tração com objetivo de se obter os valores macroscópicos do módulo de Young, razão de Poisson, resistência à tração etc. Contudo esta técnica tem alguns inconvenientes, como exemplo, a necessidade de se otimizar uma função multi-objetivo e principalmente por não levar em consideração evolução temporal do ensaio. Pensando-se neste problema e inspirado nas questões de espalhamento dinâmico em difração de elétrons, utilizouse como função objetivo o fator de confiabilidade (reliability factor) $R_{2}$ de LEED (VAN HOVE, WEINBERG, \& CHAN, 1986) definido por

$$
R_{2}=A_{2} \int\left[I_{e}(x)-c I_{c}(x)\right]^{2} d x
$$

com $A_{2}$ sendo uma constante de normalização, de maneira que todas as curvas apresentem o mesmo peso no cômputo geral. As funções $l_{e}(X)$ e $I_{c}(X)$ representam as intensidades das curvas experimentais e calculadas, respectivamente e $c=1$.

A representação geométrica do funcional dado pela Equação 9 se dá por meio dos gráficos da Figura 5 . 0 objetivo se torna minimizar a área quadrática normalizada entre as curvas experimentais e calculadas de todos os ensaios simulados de maneira que sejam as mais próximas possíveis. 

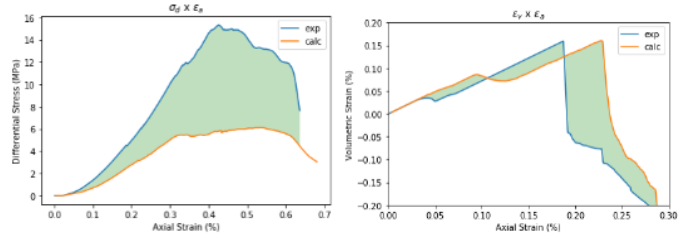

Figura 5: Exemplo da representação geométrica do funcional $R_{2}$ como a área quadrática normalizada entre as curvas experimental (em azul) e calculada (em laranja).

\section{Resultados}

Para a construção da amostra foram seguidas as sugestões da International Society of Rock Mechanics (DISCUSSION, 1999) e da American Society for Testing and Materials (ASTM International, 2014). A amostra tem a forma de um paralelepípedo reto com base quadrada de lado $44,3 \mathrm{~mm}$ e altura $88,6 \mathrm{~mm}$. É composta de esferas com raios com valores aletoriamente uniforme entre 0,056 $\mathrm{mm}$ e $1,064 \mathrm{~mm}$ de forma que a razão $d_{\max } / d_{\min }$ seja aproximadamente 2 e são representativos de um arenito turbidítico de um campo de petróleo. Com estas dimensões e geometria o número de esferas geradas foi de 38.392 e suas diferentes faces podem ser vistos em na Figura 6.

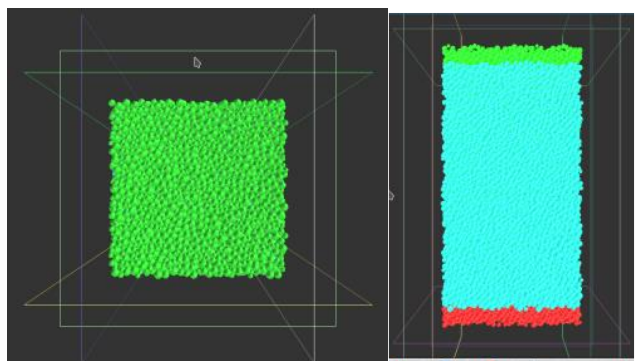

Figura 6: Amostra utilizada nos ensaios triaxiais. O centro e raio das esferas foram geradas aleatoriamente.

Foram realizados ensaios triaxiais com três tensões confinantes distintas, considerando em todos os casos $\sigma_{2}$ $=\sigma_{3}: 1 \mathrm{MPa}, 5 \mathrm{MPa}$ e $10 \mathrm{MPa}$ e a velocidade de carregamento de $0,125 \mathrm{~m} / \mathrm{s}$. Com relação ao GSA, seus parâmetros foram: $q_{V}=2,2 ; q_{A}=-5,0$ e $T_{0}=10$.

Como critério de convergência tomou-se $R_{2} \leq 0,0002$, pois isto apresenta um erro no microparâmetro $E_{m}$, em média da ordem de $0,7 \%$.

Para os casos em que não fosse atingida a convergência, foi necessária a limitação do máximo de pontos calculados. A temperatura final teve que ser em torno de 0,04 para que os sorteios fossem próximos o suficiente do mínimo global e que as variações na função objetivo fossem menores que 0,0002 .

\section{Análise de sensibilidade}

Inicialmente, foi realizada a análise da sensibilidade da função $R_{2}$ em relação aos cinco microparâmetros que compõe o modelo de contato $E_{m}, \varphi b, t, c$ e $k_{s} / k_{n}$, e os resultados podem ser vistos nas Figuras 7 e 8 .
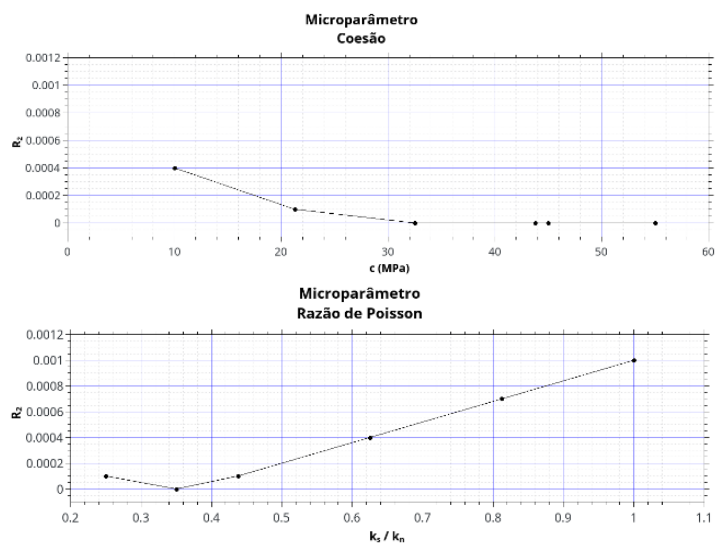

Figura 7: Análise de sensibilidade dos microparâmetros menos relevantes para a função objetivo: $c$ e $k_{s} / k_{n}$. $A$ escala do eixo $R_{2}$ varia de -0,0001 a 0,0012.
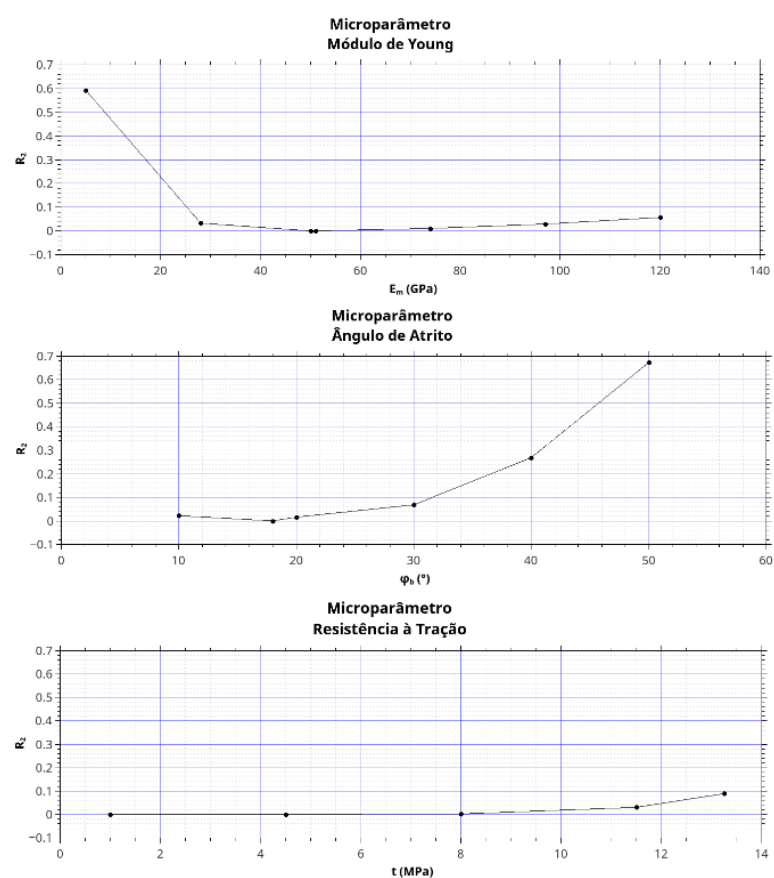

Figura 8: Análise de sensibilidade dos microparâmetros mais relevantes para a função objetivo: $E_{m}, \varphi_{b}, t$. $A$ escala do eixo $R_{2}$ varia de $-0,1$ a 0,7 .

A partir desta análise foi possível destacar que, dentro da faixa de valores permitida, as variáveis $E_{m}, \varphi_{b}, t$ se destacaram em relação $c$ e $k_{s} / k_{n}$ por possuírem uma influência significativamente maior na função objetivo. Por este motivo estas duas últimas variáveis não foram incluídas na busca.

Foi possível realizar três otimizações via GSA com a convergência sendo atingida em média após $68 \pm 47$ simulações. Os microparâmetros encontrados podem ser vistos na Tabela 1. 
Tabela 1: Microparâmetros otimizados via GSA.

\begin{tabular}{c|c|c|c|c||c}
\cline { 2 - 5 } & \multicolumn{4}{|c|}{ Otimizações } & \multicolumn{1}{c}{} \\
\hline & $\mathbf{1}$ & $\mathbf{2}$ & $\mathbf{3}$ & Média & $\begin{array}{c}\text { Erro } \\
(\boldsymbol{\%})\end{array}$ \\
\hline \hline $\left.\boldsymbol{\varphi}_{\boldsymbol{b}} \mathbf{(}^{\mathbf{}}\right)$ & 16,40 & 18,68 & 17,05 & $17 \pm 1$ & $\mathbf{5 , 5}$ \\
\hline $\boldsymbol{t}(\mathbf{M P a})$ & 7,16 & 2,21 & 6,20 & $5 \pm 3$ & $\mathbf{1 1 , 1}$ \\
\hline $\boldsymbol{E}_{\boldsymbol{m}}(\mathbf{G P a})$ & 50,20 & 49,74 & 49,59 & $49,8 \pm 0,3$ & $\mathbf{0 , 4}$ \\
\hline \hline $\boldsymbol{R}_{\mathbf{2}} \cdot \mathbf{1 0}^{-\mathbf{5}}$ & 11,03 & 2,28 & 3,77 & $5 \pm 4$ & - \\
\hline
\end{tabular}

Para o microparâmetro mais sensível, $E_{m}$, foi obtido um erro de $0,4 \%$. Já os microparâmetros $t$ e $\varphi_{b}$ foram encontrados erros de $11,1 \%$ e $5,5 \%$, respectivamente. Estes valores indicam que os microparâmetros puderam ser satisfatoriamente determinados com 0 GSA, o reduzindo consideravelmente o esforço despendido do modelador.

A resposta macroscópica, que envolve o cálculo do módulo de Young $(E)$, razão de Poisson $(v)$, ângulo de atrito interno $(\varphi)$ e resistência à compressão simples $\left(C_{0}\right)$, pode ser vista na Tabela 2 e uma das curvas de tensãodeformação original e otimizada pode ser vista na Figura 9.

Em todos os macroparâmetros calculados os erros relativos obtidos foram menores que $10 \%$. Este é outro indicativo do quanto a otimização via GSA é uma técnica eficiente e precisa.

Tabela 2: Resposta macroscópica do material simulado após a otimização.

\begin{tabular}{|c|c|c|c|c|c|}
\hline & 1 & 2 & 3 & Média & Erro \\
\hline $\begin{array}{c}E \\
(\mathrm{GPa})\end{array}$ & $24,1 \pm 0,5$ & $25,0 \pm 0,1$ & $24,1 \pm 0,4$ & $24,4 \pm 0,4$ & $1,2 \%$ \\
\hline$v$ & $0,26 \pm 0,02$ & $\begin{array}{c}0,25 \pm \\
0,02\end{array}$ & $\begin{array}{c}0,25 \pm \\
0,02\end{array}$ & $0,25 \pm 0,02$ & $<10^{-3} \%$ \\
\hline$\Phi\left({ }^{\circ}\right)$ & $63,29 \pm 0,05$ & $\begin{array}{c}50,167 \pm \\
0,002\end{array}$ & $\begin{array}{c}50.090 \pm \\
0.001\end{array}$ & $54,52 \pm 0,03$ & $8,5 \%$ \\
\hline $\begin{array}{c}C_{o} \\
(\mathrm{MPa})\end{array}$ & $0 \pm 21$ & $0 \pm 233$ & $0,2 \pm 0,1$ & $0 \pm 135$ & $<10^{-3} \%$ \\
\hline
\end{tabular}

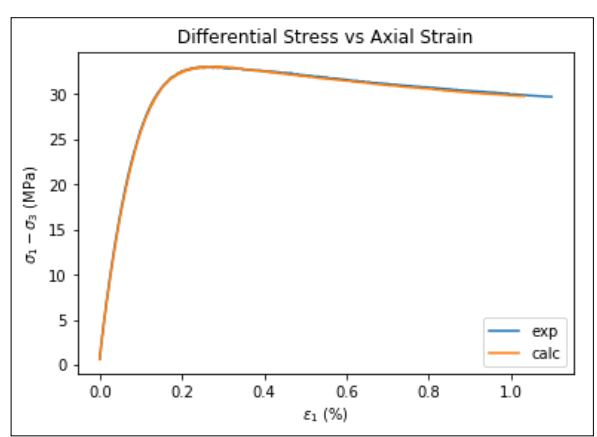

Figura 9: Comparação das curvas $\sigma_{d} \times \varepsilon_{1}$ experimental $e$ calculada após a otimização para a tensão confinante de $5 \mathrm{MPa}$.

O GSA segue um caminho aleatório no hipervolume de variáveis. Desta maneira, cada vez que é realizada uma otimização, o caminho percorrido é completamente distinto. A evolução do valor mínimo da função objetivo pode ser vista no gráfico da Figura 10 e é observado que a convergência ocorreu, na primeira otimização, após 20 reduções do valor da função objetivo, o que equivale a 122 tentativas, enquanto as otimizações seguintes encontraram a convergência após cinco reduções (em 44 tentativas) e sete reduções (em 38 tentativas).

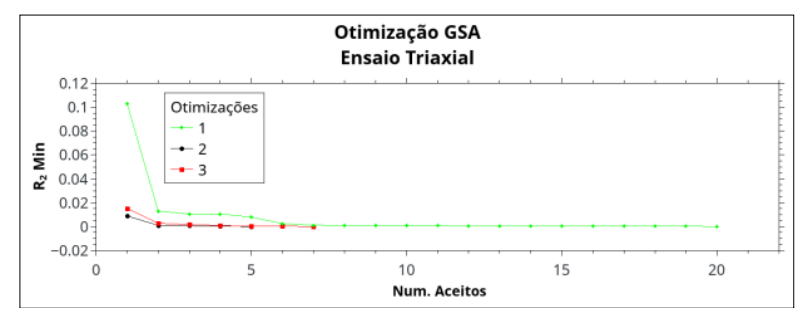

Figura 2: Evolução do valor mínimo da função objetivo nas três otimizações realizadas.

\section{Conclusões}

Neste trabalho foi utilizado o Generalized Simulated Annelealing com o intuito de calibrar os microparâmetros baseado na comparação entre as curvas experimentais e simuladas via DEM. Esta comparação foi realizada com o uso do funcional $R_{2}$ que leva em consideração a intensidade destas curvas, minimizando a área quadrática normalizada entre elas. Uma vez que a simulação dos experimentos tem como objetivo reproduzir as suas curvas, a sua resposta macroscópica será automaticamente reproduzida.

Foram simulados os ensaios triaxiais com três tensões confinantes, considerando $\sigma_{2}=\sigma_{3}: 1 \mathrm{MPa}, 5 \mathrm{MPa}$ e 10 $\mathrm{MPa}$. A partir daí foi realizada a avaliação das variáveis que influenciam nas curvas de tensão-deformação e deformações volumétrica-axial.

Dentre as variáveis que caracterizam o modelo de contato utilizado, somente três delas apresentaram sensibilidade significativa na faixa de valores que se foi permitida variar: $E_{m}, t$ e $\varphi_{b}$.

Foram realizadas três otimizações independentes, que convergiram para o mesmo ponto. Os erros relativos encontrados nos microparâmetros foram de 11,1 \% $(t), 5,5$ $\%\left(\varphi_{b}\right)$ e $0,4 \%\left(E_{m}\right)$. Para os macroparâmentros obtidos após a otimização, os erros foram de 8,5\% para o ângulo de atrito interno, 1,2 \% para o módulo de Young e menos de $10^{-3} \%$ para a razão de Poisson e resistência à compressão uniaxial.

Levando-se em conta o que foi exposto, é possível concluir que a automatização da calibração proposta neste trabalho elimina a necessidade da interação humana em cada passo, reduzindo a chance de subjetividade do modelador. Adicionalmente, devido às próprias características do GSA, este método pode ser aplicado para diferentes modelos de contato e diferentes conjuntos de simulações, a princípio sem restrições, o que torna esta aplicação bastante ampla e interessante. 


\section{Agradecimentos}

Agradecimentos especial aos colegas Guilherme Bispo, Julio Garcia, Marcus "Marquinhos" Santini e à Petrobras por esta grande oportunidade de aperfeiçoamento.

\section{Referências}

ASTM International. (2014). Standard Test Methods for Compressive Strength and Elastic Moduli of Intact Rock Core Specimens Under Varying States of Stress and Temperatures. West Conshohocken, PA. doi:https://doi.org/10.1520/D7012-14E01

CHAREYRE, B., BRIANÇOM, L., \& VILLARD, P. (2002). Theoretical versus experimental modeling of the achorage capacity of geotextitles in trenches. Geosyntetics International, 9, 97-123. doi:https://doi.org/10.1680/gein.9.0212

CHO, N., MARTIN, C., \& SEGO, D. (2007). A clumped particle model for rock. International Journal of Rock Mechanics and Mining Sciences, 44, 9971010. Fonte: http://www.sciencedirect.com/science/article/pii/ S1365160907000172

CUNDALL, P. A., \& STRACK, O. D. (1979). A discrete numerical model for granular assemblies. Géotechnique, 29, 47-65. doi:https://doi.org/10.1680/geot.1979.29.1.47

DISCUSSION. (1999). International Journal of Rock Mechanics and Mining Sciences, 36, 279-289. Fonte: http://www.sciencedirect.com/science/article/pii/ S0148906299000066

LEE, H., MOON, T., \& HAIMSON, B. C. (Apr de 2016). Borehole breakouts induced in Arkosic sandstones and a descrete element analysis. Rock Mechanics and Rock Engineering, 49, 1369-1388.

Fonte: https://doi.org/10.1007/s00603-015-0812-0

SCHOLTĖS, L., \& DONZÉ, F.-V. (2013). A DEM model for soft and hard rocks: Role of grain interlocking on strength. Journal of the mechanics and Physics of solids, 61, 352-369. Fonte: http://www.sciencedirect.com/science/article/pii/ S0022509612002268

TSALLIS, C., \& STARIOLO, D. A. (1996). Generalized simulated annealing. Physica A: Statistical Mechanics and its Applications, 233, 395-406. Fonte:

http://www.sciencedirect.com/science/article/pii/ S0378437196002713

VAN HOVE, M. A., WEINBERG, W. H., \& CHAN, C.-M. (1986). Methods of surface crystallography by LEED: Reliability factors (R-Factors). Em M. A. VAN HOVE, W. H. WEINBERG, \& C.-M. CHAN, Low-Energy Electron Diffraction: Experiment, Theory and Surface Structure Determination (pp. 237-250). Berlin: Springer Berlin Heidelberg. doi:https://doi.org;10.1007/978-3-642-82721-1_6 